\title{
Fibrolipomatous Hamartoma Arising from the Median Nerve-A Case Report
}

\author{
Aruna S. Pallewatte ${ }^{1} \quad$ Eshma C. Samarasinghe ${ }^{1}$ \\ ${ }^{1}$ Department of Radiology, National Hospital of Sri Lanka, Colombo, \\ Sri Lanka \\ Address for correspondence Dr. Aruna S. Pallewatte, Department \\ of Radiology, National Hospital of Sri Lanka, Colombo, Sri Lanka \\ (e-mail: Asp31263@hotmail.co).
}

Indian | Radiol Imaging 2021;31:472-475.

\begin{abstract}
Keywords

- coaxial cable

- fibrolipomatous hamartoma

- macrodystrophia lipomatosa

- median nerve

Fibrolipomatous hamartoma is a rare nonhereditary, congenital condition characterized by benign hyperplasia of fibroadipose tissue around nerve bundles. The median nerve is commonly affected. Underlying pathological process is mature adipose and fibrous tissue infiltrating the epineural and perineural compartments giving typical "pseudo-onion bulb" appearance on histology and "coaxial cable" appearance on magnetic resonance imaging (MRI). This case of fibrolipomatous hamartoma well illustrates its characteristic clinical, radiological, and histopathological features. Patient is a teenage female presented with painless gradually enlarging mobile lump in the central left palm. Ultrasound scan revealed a hyperechoic subcutaneous lesion, with intervening fine hypoechoic cable like structures following the course of median nerve which was thickened. Computed tomography (CT) confirmed thickened left median nerve with radiating isodense fascicles surrounded by fatty areas. There were no calcifications. MRI showed classic "coaxial cable" like T1, T2 isointense fascicles continuous with the median nerve surrounded by a fusiform lesion demonstrating fat signals on spin-echo sequences which dropped on fat-suppressed gradient echo sequences. Diagnosis of fibrolipomatous hamartoma arising from left median nerve was made, based on typical imaging findings. Biopsy confirmed fibrolipomatous hamartoma. Diagnosis can be made confidently on imaging alone especially with MRI, without the need of biopsy. Both CT and MRI demonstrate fatty mass encasing the thickened nerve fibers. On MRI, characteristic appearance is seen as T1 and T2 low intense tubular thickened neural bundles surrounded by high signal fatty tissue.
\end{abstract}

\section{Introduction}

Fibrolipomatous hamartoma is a rare nonhereditary, congenital condition characterized by benign hyperplasia of fibroadipose tissue around nerve bundles. The median nerve is commonly affected although other nerves can also be involved. This usually occurs in younger age group.
Underlying pathological process is mature adipose and fibrous tissue infiltrating the epineural and perineural compartments giving typical "pseudo-onion bulb" appearance on histology and "coaxial cable" appearance on MRI.

Here we present a case of Fibrolipomatous hamartoma which well illustrates its characteristic clinical, radiological, and histopathological features. published online July 27, 2021
DOI https://doi.org/

10.1055/s-0041-1734332 ISSN 0971-3026
(C) 2021. Indian Radiological Association.

This is an open access article published by Thieme under the terms of the Creative Commons Attribution-NonDerivative-NonCommercial-License, permitting copying and reproduction so long as the original work is given appropriate credit. Contents may not be used for commercial purposes, or adapted, remixed, transformed or built upon. (https://creativecommons.org/licenses/by-nc-nd/4.0/).

Thieme Medical and Scientific Publishers Private Ltd. A-12, Second Floor, Sector -2, NOIDA -201301, India 


\section{Clinical History}

A 17-year-old girl presented with a painless gradually enlarging lump in the central left palm approximately for 4 years duration but not associated with any other symptoms. On examination, there was a soft mobile lump over the left palm just distal to the wrist ( -Fig. 1). No evidence of sensory or motor deficit could be found in the hand. There were no finger anomalies.

\section{Imaging Findings}

Ultrasound (US) scan of the left hand and wrist revealed a hyperechoic subcutaneous lesion, with intervening fine hypoechoic cable-like structures along the course of median nerve which was thickened (-Figs. 2 and $\mathbf{3}$ ). Computed tomography $(\mathrm{CT})$ confirmed a thickened left median nerve in the carpal tunnel with radiating isodense fascicles surrounded by fatty attenuating lesion extending to the volar aspect of the hand (-Fig. 4). There were no calcifications or measurable contrast enhancement ( - Fig. 5). Magnetic resonance imaging (MRI) showed classic "coaxial cable"-like T1, $\mathrm{T} 2$ isointense fascicles continuous with the median nerve surrounded by a fusiform lesion demonstrating fat signals on spin-echo sequences which dropped on fat-suppressed gradient echo sequences (-Figs 6-9). There was no enhancement. Diagnosis of fibrolipomatous hamartoma arising from the left median nerve was made, based on these characteristic imaging findings. US-guided biopsy confirmed the radiological diagnosis of fibrolipomatous hamartoma (-Fig. 10).

\section{Discussion}

Fibrolipomatous hamartoma is a rare type of benign lesions assumed to arise congenitally due to excess fibrofatty tissue proliferation infiltrating the endoneurium, perineurium, and epineurium leading to enlargement of the affected nerve. ${ }^{1}$ Median nerve is the commonest peripheral nerve to be involved, but ulnar and radial nerves, dorsum of the

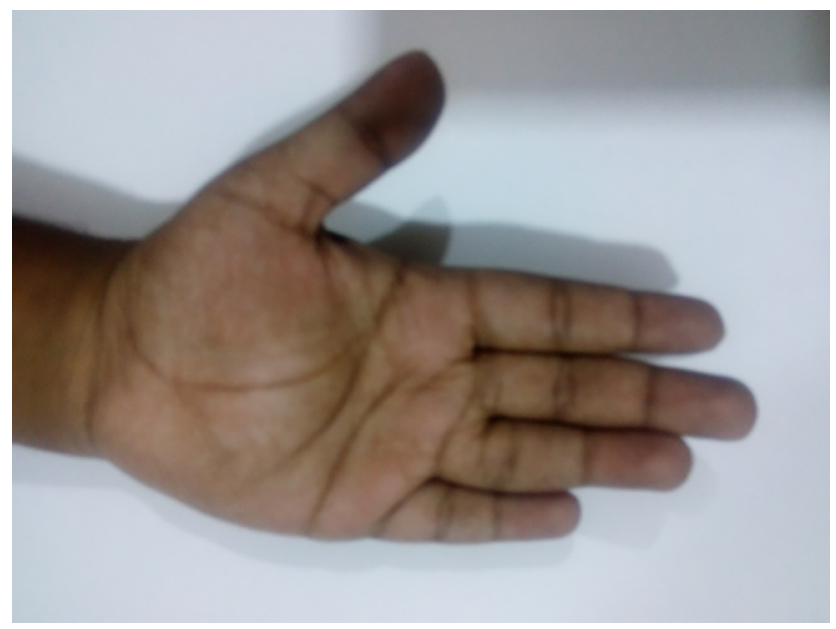

Fig. 1 Soft mobile lump over the volar aspect of the left palm just distal to the wrist.

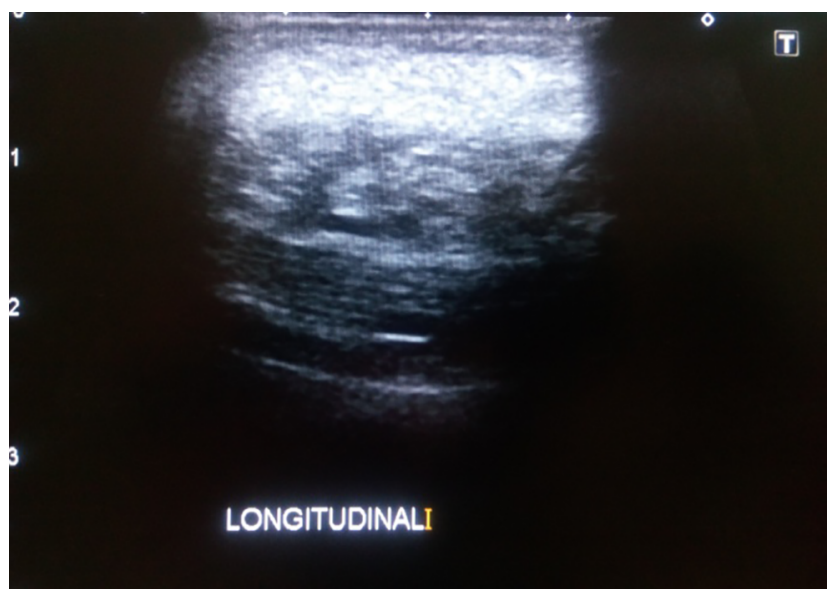

Fig. 2 Ultrasonography of the lesion revealed an echogenic mass with central linear tiny hypoechoic bands.

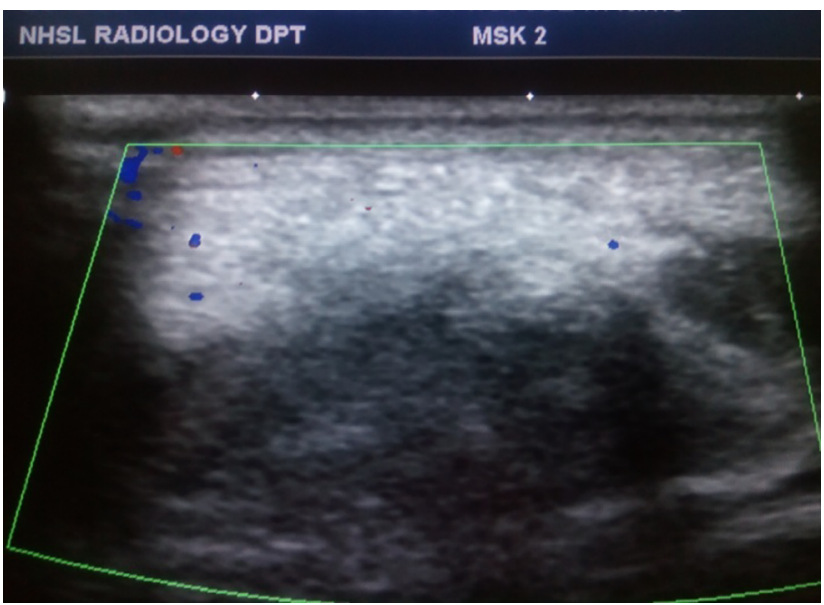

Fig. 3 Ultrasonography of the lesion revealed no demonstrable color flow on Doppler.

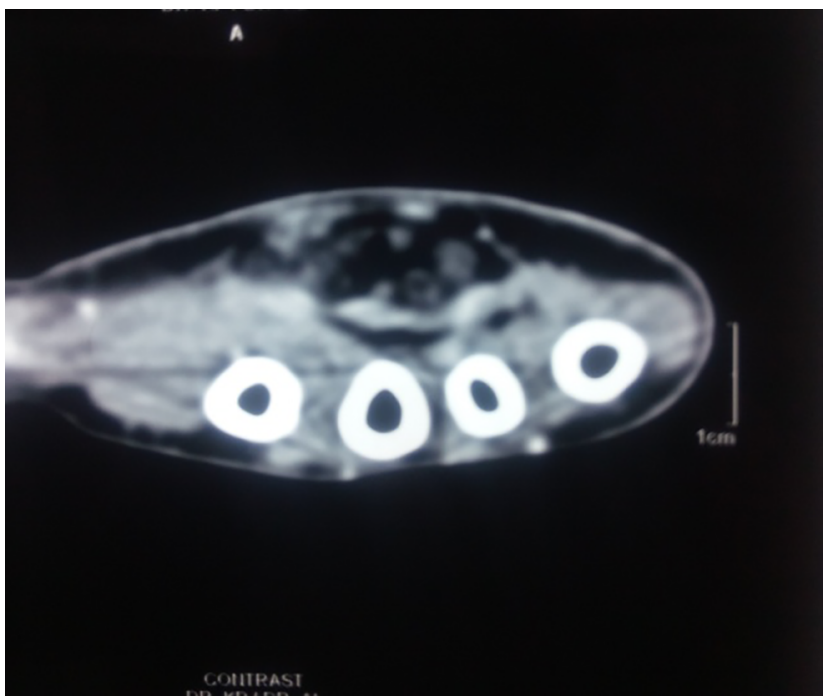

Fig. 4 Axial image of CT showing fatty lesion with central hypodense tubular structures. CT, computed tomography. 


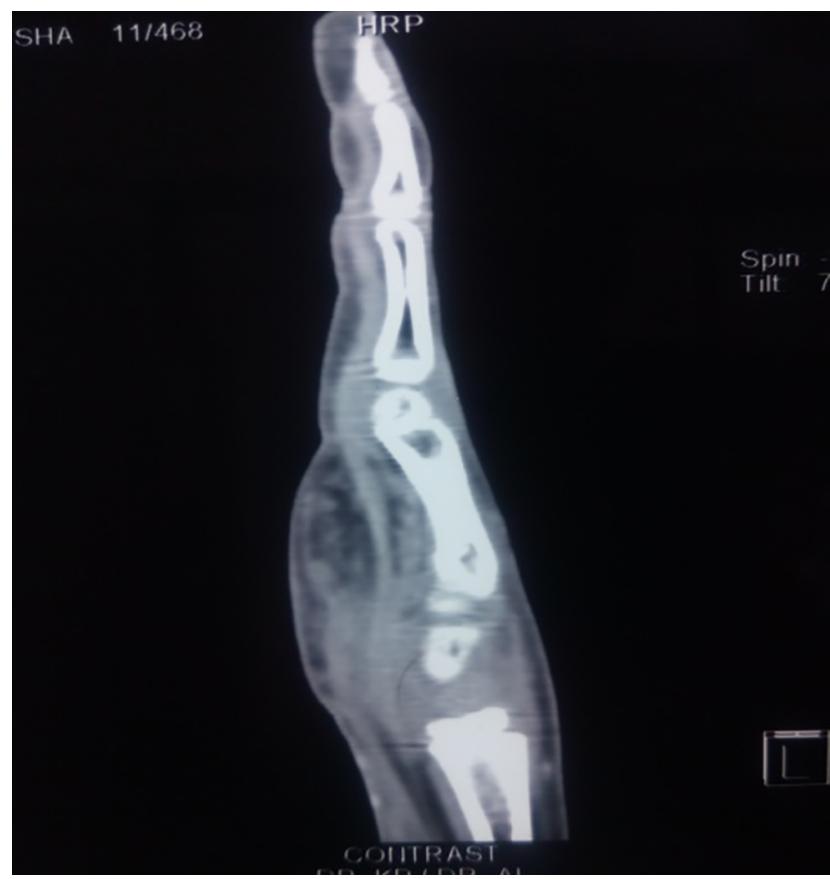

Fig. 5 Sagittal image of CT showing fatty lesion with central hypodense tubular structures. There was no enhancement of the fatty lesion. CT, computed tomography.

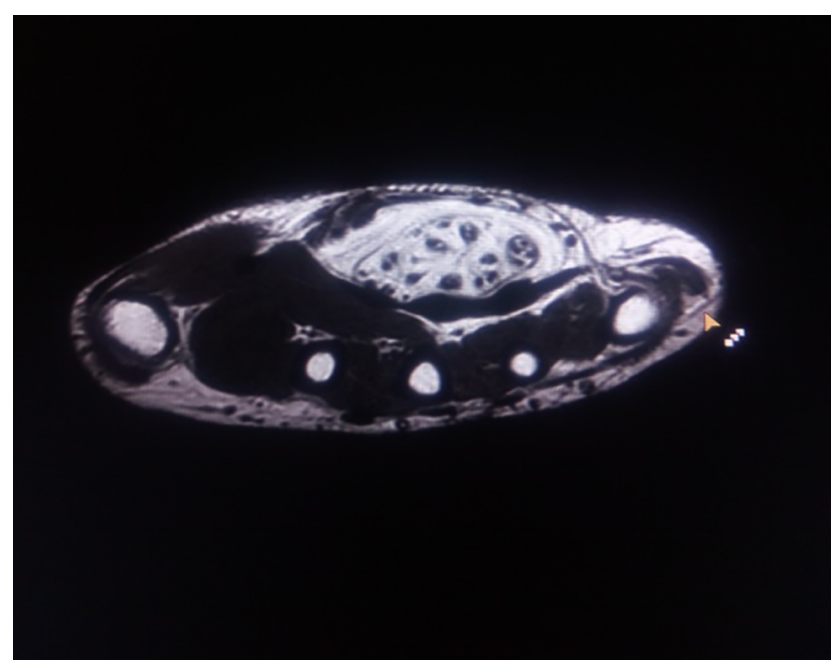

Fig. 6 Axial image of T1-weighted MRI showing classic coaxial cable-like appearance of nerve bundles with surrounding high signal fat. Magnetic resonance imaging.

foot, and brachial plexus can be affected. ${ }^{1-3}$ Fibrolipomatous hamartoma may be associated with periosteal and endosteal bony overgrowth, resulting in macrodactyly in about one-third of the cases. This variant is termed macrodystrophia lipomatosa. ${ }^{1-3}$ Usual presentation is in early adulthood with a painless lump. ${ }^{2,4}$ Rarely the condition can result pain or paresthesia due to neural compression and can present with carpal tunnel syndrome. ${ }^{4,5}$

Fibrolipomatous hamartoma is important for the clinical radiologist as the diagnosis can confidently be made on

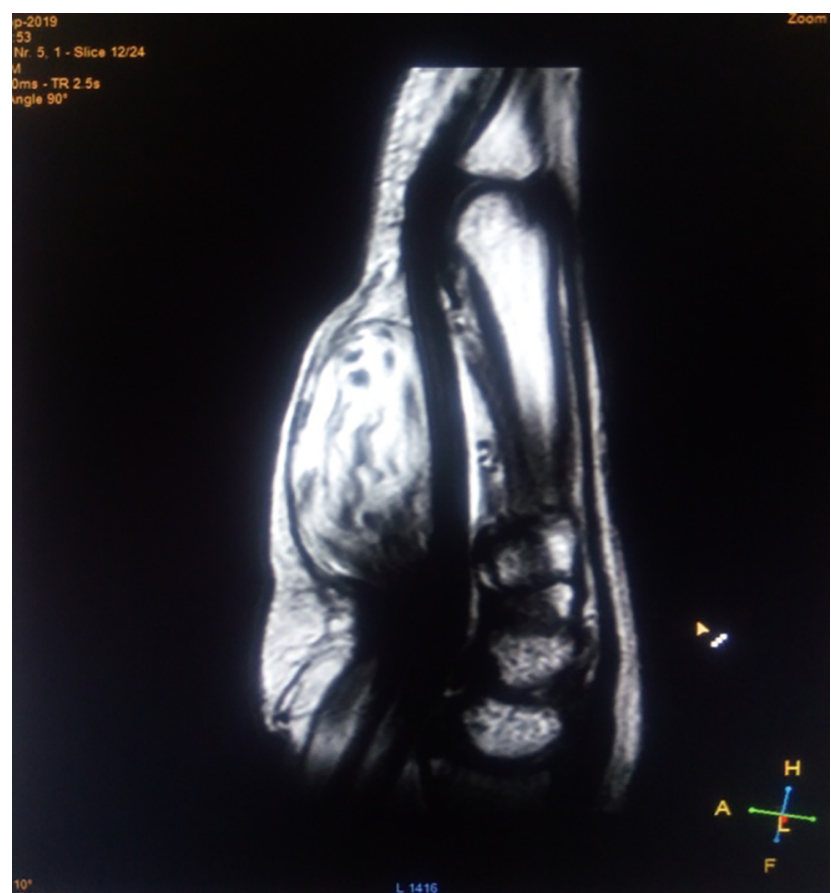

Fig. 7 Sagittal image of T1-weighted MRI showing fatty nature of the lesion. MRI, magnetic resonance imaging.

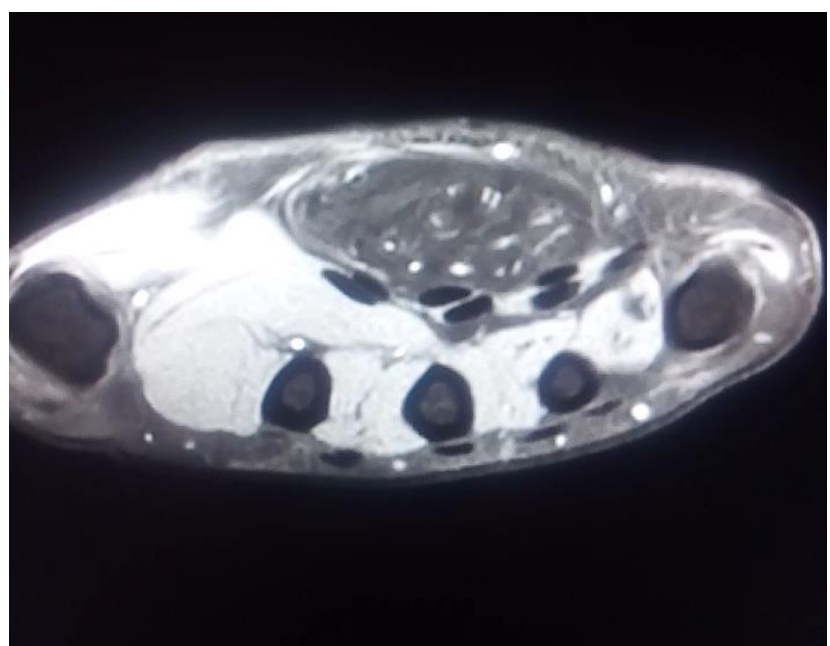

Fig. 8 Axial fat suppressed post-contrast MRI showing fatty nature of the lesion without enhancement. MRI, magnetic resonance imaging.

imaging alone especially with MRI, specially without the need of an invasive biopsy. ${ }^{3-5}$ Both CT and MRI demonstrate a fatty mass encasing the thickened nerve fibers. US provides a complementary appearance with an echogenic mass and linear hypoechoic nerve bundles in the center. ${ }^{5,6}$ On MRI, characteristic appearance of "coaxial cable" is seen as T1- and T2-low intense tubular thickened neural bundles surrounded by high signal fatty tissue..$^{1,-7}$ These lesions are preferably managed conservatively, and surgical excision is recommended only if the lesions are significantly painful or severely disfiguring.? 


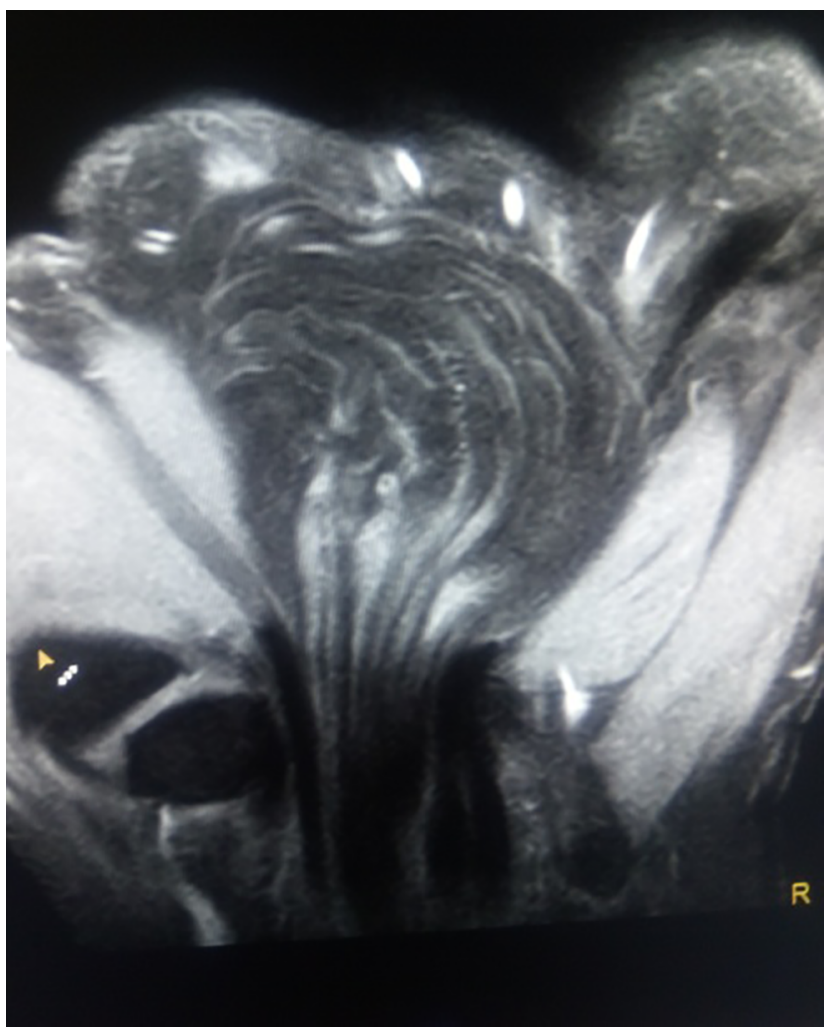

Fig. 9 Coronal fat-suppressed T1-weighted MRI image showing thickened nerve fascicles with interspersed fat. MRI, magnetic resonance imaging.

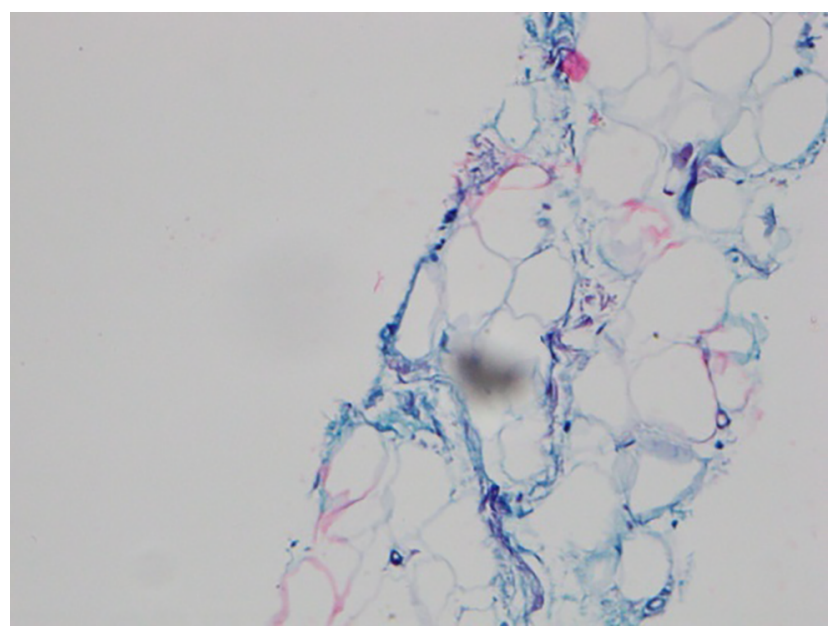

Fig. 10 Histology specimen of core biopsy demonstrating mature adipose and fibrous tissue.

\section{Conclusion}

Fibrolipomatous hamartoma commonly occurs in the younger age group and has a predilection for upper extremity nerves. The imaging features on cross-sectional imaging specially MRI are pathognomonic and may represent histopathological structure. MRI and CT are also useful in evaluating local compression and extension of the lesion in symptomatic patients. Therefore, the radiologists have a vital role in accurate noninvasive diagnosis and management of this rare hamartoma.

\section{Declaration of Patient Consent}

The authors certify that they have obtained all appropriate patient consent forms. In the form the patient(s) has/have given his/her/their consent for his/her/their images and other clinical information to be reported in the journal. The patients understand that their names and initials will not be published, and due efforts will be made to conceal their identity, but anonymity cannot be guaranteed.

\section{Financial Support and Sponsorship}

None.

\section{Conflict of Interest}

None declared.

\section{References}

1 Gilet A, Jeremy M, Gould E. Fibrolipomatous hamartoma of the median nerve. Radiol Case Rep 2015;3(3):195

2 Toms AP, Anastakis D, Bleakney RR, Marshall TJ. Lipofibromatous hamartoma of the upper extremity: a review of the radiologic findings for 15 patients. AJR Am J Roentgenol 2006;186(3):805-811

3 Azeemuddin M, Waheed AA, Khan N, Sayani R, Ahmed A. Fibrolipomatous hamartoma of the median nerve with macrodystrophia lipomatosa. Cureus 2018;10(3):e2293

4 So CK, Tam KF, Lui CY, Lee CM. Fibrolipomatous hamartoma of the median nerve. J HK Coll Radiol 2007;10:81-84

5 Malan L, Bezuidenhout AF, Banderker E. Fibrolipomatous hamartoma of the median nerve. S Afr J Rad 2015;19Article. \#886, 3 pages

6 Diwakar N, Kamble RB, Gidaganti M. Imaging of neurofibrolipoma of median nerve. Neurol India 2011;59(3):488-489

7 Razzaghi A, Anastakis DJ. Lipofibromatous hamartoma: review of early diagnosis and treatment. Can J Surg 2005;48(5):394-399 